\title{
Integrating motivational interviewing with cognitive behavioural therapy for anxiety disorders, depression and co-morbid unhealthy lifestyle behaviours: a randomised controlled pilot trial
}

\author{
Ata Ghaderi ${ }^{1}$ (D) Ingvar Rosendahl ${ }^{2}$ and Benjamin Bohman ${ }^{2 \star(D)}$ \\ ${ }^{1}$ Division of Psychology, Department of Clinical Neuroscience, Karolinska Institutet, SE-171 77, Stockholm, Sweden and \\ ${ }^{2}$ Centre for Psychiatry Research, Department of Clinical Neuroscience, Karolinska Institutet and Stockholm Health Care \\ Services, Region Stockholm, SE-113 64, Stockholm, Sweden \\ *Corresponding author. Email: benjamin.bohman@ki.se
}

(Received 19 February 2021; revised 15 June 2021; accepted 15 July 2021; first published online 19 August 2021)

\begin{abstract}
Background: A substantial proportion of patients receiving cognitive behavioural therapy (CBT) do not achieve remission, and drop-out is considerable. Motivational interviewing (MI) may influence nonresponse and drop-out. Previous research shows that MI as a pre-treatment to CBT produces moderate effects compared with CBT alone. Studies integrating MI with CBT (MI-CBT) are scarce.

Aims: To test the feasibility of MI-CBT in terms of therapist competence in MI and various participant measures, including recruitment and retention. In addition, separate preliminary evaluations were conducted, exploring the effects of CBT alone for anxiety disorders and depression, and of MI-CBT for anxiety disorders, depression and unhealthy lifestyle behaviours.

Method: Using a randomised controlled parallel trial design, participants were recruited in routine psychiatric care and allocated to CBT alone or MI-CBT. Means in feasibility measures and withincondition Hedges' $g$ effect sizes in treatment outcome measures were calculated. Authors were not blind to treatment allocation, while independent raters were blind.

Results: Seventy-three patients were assessed for eligibility, and 49 were included. Participant perceptions of treatment credibility, expectancy for improvement, and working alliance were similar for both conditions. Overall, effect sizes were large across outcome measures for both conditions, including anxiety and depressive symptoms and functional impairment. However, therapists did not acquire sufficient competence in MI and the drop-out rate was high.

Conclusions: MI-CBT proved feasible in some respects, but the present study did not support the progression to a randomised controlled trial designed to assess the effectiveness of MI-CBT. Additional pilot studies are needed.
\end{abstract}

Keywords: anxiety disorders; cognitive behavioural therapy; depression; motivational interviewing; unhealthy lifestyle behaviours

\section{Introduction}

Cognitive behavioural therapy (CBT) is probably an efficacious treatment for anxiety disorders and depression, taking into account publication bias, the quality of trials, and the influence of control conditions (Cuijpers et al., 2016). However, only half of patients with an anxiety disorder achieve

(C) The Author(s), 2021. Published by Cambridge University Press on behalf of the British Association for Behavioural and Cognitive Psychotherapies. This is an Open Access article, distributed under the terms of the Creative Commons Attribution licence (http:// creativecommons.org/licenses/by/4.0/), which permits unrestricted re-use, distribution, and reproduction in any medium, provided the original work is properly cited. 
remission status post-treatment (Springer et al., 2018) and every fifth patient with anxiety, and every third patient with depression, drops out of treatment (Fernandez et al., 2015).

Motivational interviewing (MI) is a collaborative conversation style for strengthening an individual's motivation and commitment for behaviour change (Miller and Rollnick, 2013). MI may be useful for enhancing CBT because insufficient motivation, often expressed as ambivalence about or resistance to change, is a patient factor that may influence non-response and drop-out (Miller and Rollnick, 2013; Westra, 2012). MI has been employed as an adjunct to CBT in several studies, and a recent meta-analysis including 12 studies of adult patients with anxiety disorders showed that the combination had a moderate effect on symptom reduction compared with CBT alone (Marker and Norton, 2018). Of the published studies included in the meta-analysis, all used MI as a prelude to CBT, whereas only two studies incorporated additional MI principles throughout treatment, that is, integrated MI and CBT (MI-CBT). Simpson and colleagues (Simpson et al., 2010) found no differences in symptom improvement or adherence to CBT procedures in a small sample of participants with obsessive-compulsive disorder (OCD) who were recruited in a research clinic setting and randomly allocated to CBT alone or MI-CBT. One explanation offered by the authors was that the dose or delivery of MI was sub-optimal, because an MI module was available for use if participants showed signs of decreased motivation only if standard CBT procedures for making participants adhere to treatment were ineffective (Simpson et al., 2010). The authors suggested that a more integrated protocol might have yielded different results. In a wellcontrolled and adequately powered randomised trial, Westra and colleagues (Westra et al., 2016) found that at post-assessment there were no differences in outcomes in participants with generalised anxiety disorder (GAD) who were recruited from the general population and received CBT alone or MI-CBT. However, over follow-up assessments at 6 and 12 months, participants in the MI-CBT condition had benefited more in terms of reduction in worry and general distress. Thus, there are only two studies of MI-CBT for anxiety disorders in adults and none was conducted in routine psychiatric care using regular therapists. There is a paucity of studies of combinations of MI and CBT for depression as the principal diagnosis. This is unfortunate considering that decreased motivation is a significant problem in depression.

Unhealthy lifestyle behaviours related to diet, alcohol use, physical activity or tobacco use are more prevalent in individuals with anxiety disorders and depression than in the general population (e.g. Fluharty et al., 2017; Zschucke et al., 2013). Unhealthy lifestyle behaviours not only contribute to additional suffering, but also increase the risk of chronic medical conditions and premature mortality (e.g. Batelaan et al., 2016). Both MI and CBT are effective in improving lifestyle behaviours and preventing disease (e.g. National Board of Health and Welfare, 2018) and the integration of the treatments may provide additional benefits. Considering the high co-morbidity of unhealthy lifestyle behaviours with psychiatric disorders, a potentially useful approach is to focus on both of these conditions in the same treatment using MI-CBT.

Pilot studies are appropriate if there is uncertainty about future randomised controlled trial (RCT) feasibility (Eldridge et al., 2016). The primary aim of the present study was to investigate the feasibility of MI-CBT in preparation for a future RCT designed to assess the effectiveness of MI-CBT in routine psychiatric care. Because there are only two previous studies on MI-CBT for anxiety disorders, there are several uncertainties concerning future RCT feasibility, including recruitment and retention of participants in routine psychiatric care. In addition, feasibility was investigated in terms of therapist competence in MI following a brief training, and participant perceptions of treatment credibility, expectancy for improvement, and working alliance between participants and therapists. Support for progression to an RCT was deemed sufficient if it was able to recruit a reasonable number of participants, they remained in the study, and therapists acquired competence in MI. A secondary aim of the present study was to conduct separate preliminary evaluations exploring 
the effects of CBT alone for anxiety disorders and depression and of MI-CBT for anxiety disorders, depression, and co-morbid unhealthy lifestyle behaviours. For both treatments, psychiatric symptoms, unhealthy lifestyle behaviours, functional impairment, and quality of life were used as outcome measures. It was expected that MI-CBT would be as feasible as CBT in the above respects and that both conditions would show clinically relevant improvements in outcome measures.

\section{Method \\ Design}

The present pilot trial had a randomised controlled parallel design and was conducted between 2013 and 2015 at a psychiatric clinic specialised in CBT for anxiety disorders and depression in Stockholm, Sweden. Participants were consecutively included in the study and provided treatment. Randomisation was conducted on a 1:1 basis using an online service (www.random.org).

\section{Participants}

Inclusion criteria were: (a) 18 years of age or older, (b) a principal diagnosis according to the Diagnostic and Statistical Manual of Mental Disorders (4th edition, text revision: DSM-IV-TR; American Psychiatric Association, 2000) of panic disorder, social anxiety disorder (SAD), OCD, GAD, post-traumatic stress disorder (PTSD), or major depressive disorder, and (c) at least one of the following unhealthy lifestyle behaviours: unhealthy eating habits, hazardous use of alcohol, insufficient physical activity, or tobacco use, according to criteria suggested by the National Board of Health and Welfare (2011). Exclusion criteria were: (a) DSM-IV-TR criteria for psychotic symptoms, (b) $D S M-I V$-TR criteria for substance abuse other than alcohol, (c) DSM-IV-TR criteria for substance dependence including alcohol, (d) moderate to severe suicide risk according to the Mini-International Neuropsychiatric Interview (MINI; Sheehan et al., 1998) or a score of 2 or above on item 9 on the Patient Health Questionnaire-9 (PHQ-9; Kroenke et al., 2001), and (e) another simultaneous psychological treatment. Participants were recruited by the therapist who conducted diagnostic assessment. The last author assigned participants to treatment.

\section{Treatments}

\section{Cognitive behavioural therapy}

Disorder-specific CBT protocols were used in both the CBT and MI-CBT conditions, including panic control treatment (Craske and Barlow, 2008) for panic disorder, CBT (Turk et al., 2008) for SAD, exposure with response prevention (Franklin and Foa, 2008) for OCD, intolerance of uncertainty therapy (Dugas and Robichaud, 2007) for GAD, prolonged exposure (Foa et al., 2007) for PTSD, and behavioural activation (Martell et al., 2010) for depression. To be consistent with routine practice, CBT did not involve a particular focus on unhealthy lifestyle behaviours; rather, therapists were instructed to target such behaviours only to the extent they typically do when practising CBT for psychiatric disorders. Sessions in the CBT condition were conducted on a weekly basis and were typically 45 minutes in length. During the treatment period, therapists in both conditions received supervision in CBT by independent supervisors every third week as part of routine practice. 
model was developed in discussions between the authors only. Participants were treated within an MI framework in accordance with the four processes characterising MI (Miller and Rollnick, 2013): (1) engaging patients in a mutually trusting and respectful working relationship, (2) focusing on goals for treatment, (3) evoking and strengthening motivation for behaviour change, and (4) planning and implementing treatment. These processes are overlapping and both sequential and recursive: earlier processes lay the foundation for later processes and continue to run beneath them, and treatment may momentarily return to an earlier process that requires renewed attention (Miller and Rollnick, 2013).

The MI-CBT model was compiled in the form of a treatment guide, designed to be used flexibly depending on the individual participant's motivational state and whether therapist and participant agreed on first to focus on the psychiatric disorder and then to focus on the unhealthy lifestyle behaviour, or vice versa. A third option was to focus on the psychiatric disorder and the lifestyle behaviour simultaneously, as the problems for some patients are functionally related (e.g. alcohol use as a safety behaviour in SAD). In the treatment guide, the first three processes are covered in the first one to three sessions (depending on the patient's motivational state) and consist of pure MI, whereas the fourth process consists of CBT integrated with MI. Psychiatric disorders were treated according to the CBT protocols and unhealthy lifestyle behaviours were targeted using generic CBT principles and strategies (e.g. functional analysis or case conceptualisation, thought records, stimulus control). Thus, in the treatment guide, the planning and implementation process is the most comprehensive. Planning and implementation are conducted within an MI framework; for example, sessions are client-centred and conducted in an 'MI sprit', and patient autonomy is emphasised. Although CBT is at the core of this process, MI principles and strategies are employed whenever patient motivation decreases; on such occasions, treatment may momentarily return to an earlier process. Examples include sub-optimal engagement in exposure (return to evoking) and ruptures in the working alliance (return to engaging). Thus, in contrast to CBT protocols, the MI-CBT guide puts an emphasis on resolving ambivalence and strengthening motivation whenever ambivalence appears, to facilitate behaviour change in anxiety or depression as well as lifestyle behaviours, and to increase adherence to CBT procedures. As in the CBT condition, sessions in the MI-CBT condition were conducted on a weekly basis and were typically 45 minutes in length. During the treatment period, therapists in the MI-CBT condition had access to regular supervision in MI-CBT by the last author.

\section{Therapists}

Six clinical psychologists specialising in CBT were randomly allocated to practice CBT $(n=3)$ or MI-CBT $(n=3)$. In the CBT condition, one therapist was female, mean age was 31.0 years (range 30-32), mean number of years of clinical experience was 1.3 (range 0-3), two therapists had previous training in MI, and the mean score of competence in CBT on the Cognitive Therapy Scale (CTS; www.beckinstitute.org) was 43.3 (range 30-52). In the MI-CBT condition, two therapists were females, mean age was 39.0 years (range 37-40), mean number of years of clinical experience was 4.7 (range $0-8$ ), one therapist had previous training in MI, and the mean score of competence in CBT was 47.0 (range 37-57).

\section{Training}

Therapists in the MI-CBT condition received training in MI. Training was conducted according to research findings of what is required for acquisition of competence in MI; that is, a combination of learning methods, including systematic feedback on performance and supervision (Madson et al., 2009; Miller et al., 2004). The MI training included five occasions of didactic presentation, a 1-day skills training, systematic feedback on MI performance based on one session coded according to 
the Motivational Interviewing Treatment Integrity (MITI; Moyers et al., 2010), and four supervision occasions with an independent supervisor who was a member of the Motivational Interviewing Network of Trainers. In addition, therapists in the MI-CBT condition were provided with instructions on how to implement MI-CBT in their practice.

\section{Assessment}

Measures used in the present study are well-established and have good psychometric properties. Unless otherwise specified, assessments in participants were administered before and after treatment and 6 months following treatment completion, and higher scores indicate more severe symptoms or unhealthy lifestyle behaviours. All assessments in participants were webbased, except for diagnosis, therapist competence in CBT and MI, and observer-assessed participant motivation for behaviour change. The web-based assessment system included several reminders administered automatically via email and short message service. Upon nonresponse following reminders, participants were contacted by telephone. Authors were not blind to treatment allocation, while independent raters of competence in CBT and observerassessed participant motivation were blind. Unless otherwise stated, internal consistency of measures using Cronbach's $\alpha$ was calculated for the entire sample at pre-assessment.

\section{Therapist competence in cognitive behavioural therapy}

Competence in CBT was assessed in therapists in both conditions at the beginning of the treatment period using the CTS. The CTS consists of 11 items, which are rated between 0 and 6. Competence was examined in audio-recorded sessions with patients not participating in the present study. Therapists were instructed to select one patient with whom they judged they could perform as good CBT as possible (assumed to reflect 'best practice'). A commonly referred to, although not validated, threshold for competence on the CTS is 39 points (Muse and McManus, 2013). One therapist in each condition did not reach this threshold.

\section{Principal diagnosis}

The MINI was used to determine a principal diagnosis of an anxiety disorder or depression. Typically, diagnostic assessment was conducted by a therapist other than the one who conducted treatment.

\section{Unhealthy lifestyle behaviours - screening}

Definitions of unhealthy lifestyle behaviours suggested by the National Board of Health and Welfare (2011) were used and the Board's screening items were administered to all potential participants to determine study eligibility. Unhealthy eating habits were assessed using four items measuring the frequency of consumption of some healthy and unhealthy foods. Hazardous use of alcohol was assessed using two items measuring the frequency and amount of consumption, corresponding to items 2 and 3 of the Alcohol Use Disorders Identification Test (AUDIT; Saunders et al., 1993). Insufficient physical activity was defined as less than 150 minutes of moderate physical activity or less than 75 minutes of intensive physical activity or less than 150 minutes per week of both in combination. Tobacco use was defined as daily smoking or use of snuff.

\section{Feasibility}

To assess competence in MI in therapists providing MI-CBT, the MITI version 3.1.1 (Moyers et al., 2010) was used. The MITI consists of 12 items, which yield six indicators of competence: MI spirit, empathy, per cent complex reflections, per cent open questions, 
reflection-to-question ratio, and per cent MI-adherent behaviours, each with specified thresholds for beginning proficiency proposed by Moyers and colleagues (Moyers et al., 2010). Competence in MI was examined in one audio-recorded session with a participant per therapist. Sessions were coded by an independent rater demonstrating 'excellent' inter-rater reliability [intra-class coefficient (ICC) $=0.75-1.00$ ] on five items, 'good' (ICC $=0.60-0.74)$ on four items, and 'fair' (ICC $=0.40-0.59)$ on three items compared with a 'gold standard' rater, according to a classification system proposed by Cicchetti (1994).

The Credibility/Expectancy Questionnaire (CEQ; Devilly and Borkovec, 2000) was used to assess treatment credibility and expectancy for improvement. The CEQ consists of six items on two separate subscales and was administered following the third session, with higher scores indicating higher credibility $(\alpha=0.86)$ or expectancy $(\alpha=0.87)$. As responses are made on different scales (1-9 and $0-100 \%)$, a composite score is derived for each subscale by first standardising individual items and then summing those items for each subscale.

The Working Alliance Inventory - Short Revised (WAI-SR; Hatcher and Gillaspy, 2006) consists of 12 items and was used to assess participant-reported treatment alliance. The WAISR was administered following the sixth session, with higher scores indicating stronger working alliance $(\alpha=0.95)$.

\section{Treatment effects}

Psychiatric disorder-specific symptoms. The Panic Disorder Severity Scale - Self-Rated (Houck et al., 2002) was used to assess panic symptoms, the Liebowitz Social Anxiety Scale, SelfReport (Fresco et al., 2001) for social anxiety symptoms, the Yale-Brown Obsessive Compulsive Scale Self-Report (Baer et al., 1993) for obsessive-compulsive symptoms, the Penn State Worry Questionnaire (PSWQ; Meyer et al., 1990) for worry symptoms, and the Posttraumatic Diagnostic Scale (Foa et al., 1997) to assess post-traumatic stress symptoms. These measures were administered to participants depending on the principal diagnosis; for example, the PSWQ was administered to participants with GAD.

Unhealthy lifestyle behaviours. Unhealthy eating habits were measured using six items proposed by the National Food Agency (Sepp et al., 2004) to assess the frequency of consumption of certain unhealthy foods indicative of diet quality. The AUDIT consists of 10 items and was used to assess alcohol use. The International Physical Activity Questionnaire (IPAQ; Craig et al., 2003) was used to assess physical activity. The IPAQ assesses physical activity in terms of metabolic equivalent of task (MET), a measure of energy expenditure. The IPAQ provides a measure in MET minutes of how much time per week is spent on three activity levels: moderate walks, moderately vigorous physical activity, and very vigorous physical activity. Higher scores indicate more physical activity. Tobacco use was assessed by items concerning number of cigarettes per day or boxes of snuff per week as proposed by the National Board of Health and Welfare (2011). These measures were administered to participants depending on which unhealthy lifestyle behaviours they showed; for example, the IPAQ was administered to participants with insufficient physical activity.

Other measures. The Generalized Anxiety Disorder Assessment 7 (GAD-7; Spitzer et al., 2006) consists of seven items and was used to assess general anxiety symptoms $(\alpha=0.89)$ and the PHQ-9 (Kroenke et al., 2001), which consists of nine items, was used to assess depressive symptoms $(\alpha=0.78)$. The Sheehan Disability Scale (SDS; Sheehan, 1983) consists of three items and was used to assesses functional impairment, with higher scores indicating more functional impairment $(\alpha=0.64)$. The Quality of Life Inventory (QOLI; Frisch et al., 1992) consists of 32 items and was used to assess quality of life, with higher scores indicating higher quality $(\alpha=0.79)$.

Several measures were used to assess potential mediators of effects to investigate in a future RCT. The Change Questionnaire (CQ; Miller and Johnson, 2008) consists of three items and 
was used to assess participant-reported motivation for behaviour change. Two versions of the CQ were developed: one for the psychiatric disorder $(\alpha=0.33)$ and one for the lifestyle behaviour $(\alpha=0.46)$. A self-efficacy (SE) measure was developed for use in the present study based on guidelines for SE scale construction (Bandura, 2006) and previous personal experience of SE scale development (e.g. Bohman, Rasmussen, \& Ghaderi, 2016). Two versions were developed: one for the psychiatric disorder (six items; $\alpha=0.77$ ) and one for the lifestyle behaviour (five items; $\alpha=0.72$ ). Items concerned efficacy beliefs in performing activities or being in situations despite psychiatric symptoms or lifestyle behaviours and were rated between 0 and 10. To test multiple administration in preparation for a future RCT, the CQ and the SE measure were administered following the third and sixth session; the SE scale was also administered at preassessment. The Client Language Assessment in Motivational Interviewing (Miller et al., 2007) was used to measure observer-assessed participant motivation for behaviour change in terms of change talk; that is, utterances that are in favour of change, as opposed to sustain talk; utterances that are in favour of the status quo. Per cent change talk [change talk/(change talk + sustain talk)] was assessed based on three self-selected audio-recordings of the first or second session per therapist.

\section{Data analysis}

Data were analysed using the SPSS (version 26, SPSS Inc., Chicago, IL, USA) and an online effect size calculator (https://effect-size-calculator.herokuapp.com). Differences in the number of sessions between the CBT and MI-CBT conditions were examined using an independent samples $t$-test and in the proportion of drop-out between the conditions using relative risk with 95\% confidence intervals (CI). Because participants had one of several psychiatric disorders as the principal diagnosis and there were few participants per diagnosis, a psychiatric symptoms index measure was created using the standardised scores of the symptoms measure of the principal diagnosis for each participant. The same procedure was used to create an unhealthy lifestyle behaviours index measure based on measures of unhealthy diet, alcohol use, physical activity (with scores reversed), and tobacco use. Withincondition effect sizes were calculated for feasibility measures and for treatment outcome measures from pre- to post-assessment and from pre- to 6-month follow-up assessment. Equations for within-condition Cohen's $d$ effect sizes suggested by Lakens (2013) and employed in the online effect size calculator with Hedges' correction $g$ were used.

\section{Results}

\section{Feasibility}

Seventy-three patients were assessed for eligibility during an 8-month period; of these, 24 were excluded. Thus, 49 participants were randomly allocated to CBT alone $(n=21)$ or MI-CBT $(n=28)$. For participant flow through the study, including reasons for exclusion, see Fig. 1 . In the CBT condition, $14(66.7 \%)$ participants were females, mean age was 40.3 years $(S D=13.2)$, and $18(85.7 \%)$ were born in Sweden. In the MI-CBT condition, $16(57.1 \%)$ participants were females, mean age was 41.7 years $(S D=13.4)$, and $27(96.4 \%)$ were born in Sweden. For principal psychiatric diagnosis and unhealthy lifestyle behaviours in participants, see Table 1.

Mean number of treatment sessions was 8.2 sessions $(S D=5.2)$ in the CBT condition, and 10.7 sessions $(S D=7.7)$ in the MI-CBT condition, a difference that was not statistically significant, $t_{47}=-1.276, p=.208$. Twenty-one (42.9\%) participants dropped out of treatment, 11 (52.4\%) in the CBT condition and 10 (35.7\%) in the MI-CBT condition; the proportion of drop-out between conditions was not statistically different, relative risk $=1.44[0.76,2.75]$ 


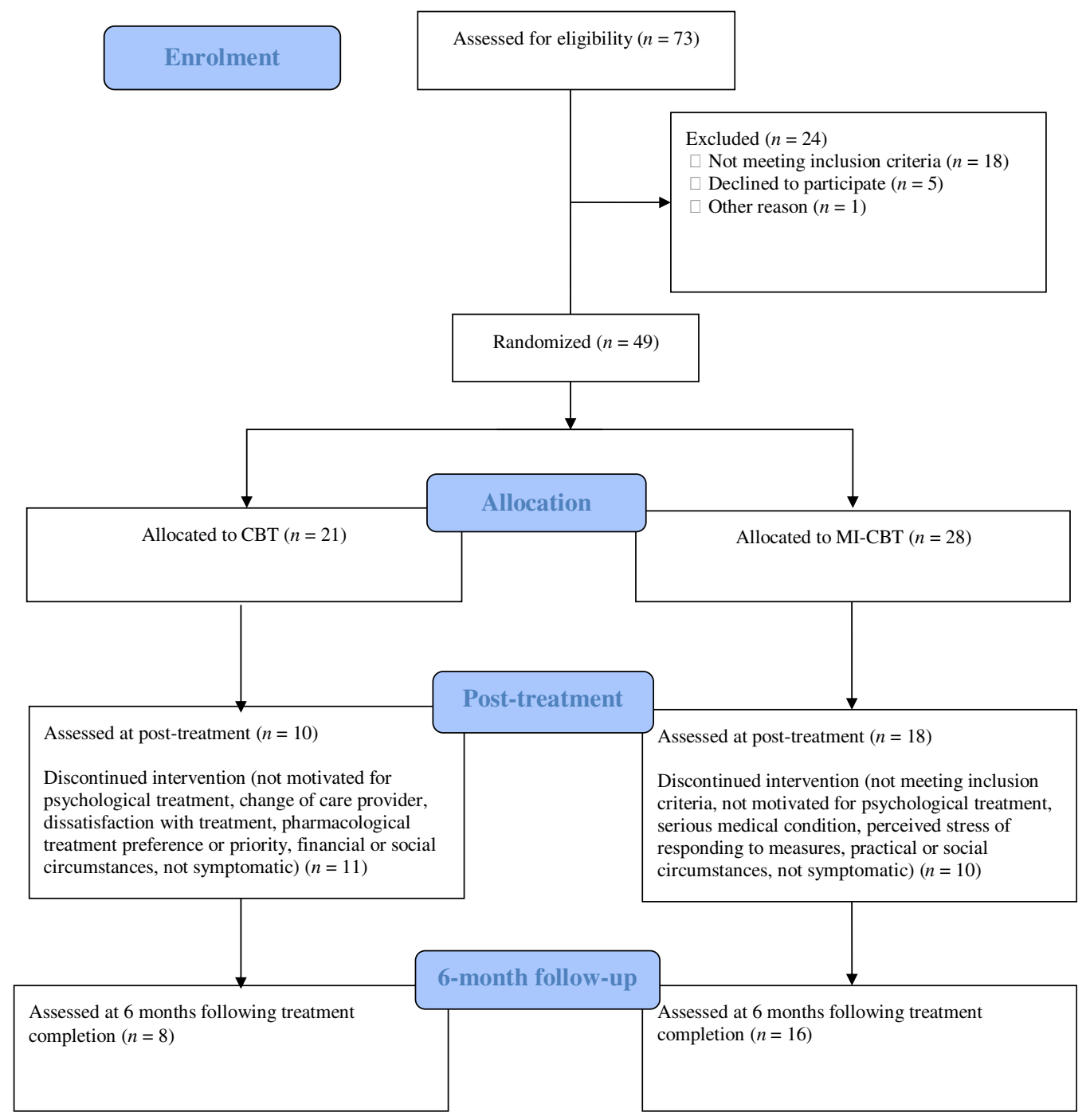

Figure 1. Participant flow through the study. CBT, cognitive behavioural therapy; MI, motivational interviewing.

(indicated by CI overlapping 1). Missing values across variables (i.e. feasibility and treatment outcome measures at different assessments) ranged from one to three missing values. In the MI-CBT condition, one therapist reached beginning proficiency in MI on four of the six MITI indicators of competence, and two therapists on two of the indicators. Means in participant perceptions of treatment credibility, expectancy for improvement, and working alliance were similar across the conditions; see Table 2. No adverse events were observed in the participants.

\section{Treatment effects}

Means and within-condition Hedges' $g$ effect sizes in treatment outcome measures across assessments are presented in Table 3. Means changed in the expected direction across assessments for both the CBT and MI-CBT conditions, except for in the unhealthy lifestyle behaviours index for both conditions. Specifically, mean scores decreased in the psychiatric symptoms index, GAD-7, PHQ-9 
Table 1. Principal psychiatric diagnosis and unhealthy lifestyle behaviours

\begin{tabular}{lrr}
\hline & CBT $n(\%)$ & MI-CBT $n$ (\%) \\
\hline Principal diagnosis & & $17(60.7)$ \\
Anxiety disorder & $9(42.9)$ & $11(39.3)$ \\
Depression & $12(57.1)$ & $19(67.9)$ \\
Unhealthy lifestyle behaviour & $11(52.4)$ & $17(60.7)$ \\
Unhealthy eating & $11(52.4)$ & $11(39.3)$ \\
Hazardous alcohol use & $9(42.9)$ & $11(39.3)$ \\
Insufficient physical activity & $7(33.3)$ & $2.0(0.9)$ \\
Tobacco use & $1.8(0.7)$ & \\
Number of unhealthy lifestyle behaviours, mean (SD) & & \\
\hline
\end{tabular}

CBT, cognitive behavioural therapy; MI, motivational interviewing.

Table 2. Means in treatment credibility, expectancy for improvement, and working alliance

\begin{tabular}{llll}
\hline & Condition & Session 3 & Session 6 \\
\hline \multirow{2}{*}{ CEQ credibility } & CBT & Mean $=-0.21, S D=2.57, n=16$ & \\
& MI-CBT & Mean $=0.16, S D=2.78, n=21$ & \\
CEQ expectancy & CBT & Mean $=-0.05, S D=2.82, n=16$ & \\
& MI-CBT & Mean $=0.04, S D=2.62, n=21$ & Mean $=63.38, S D=14.60, n=13$ \\
WAI-SR & CBT & & Mean $=62.29, S D=14.56, n=17$ \\
& MI-CBT & & \\
\hline
\end{tabular}

CBT, cognitive behavioural therapy; MI, motivational interviewing; CEQ credibility, Credibility/Expectancy Questionnaire, treatment credibility subscale (standardised); CEQ expectancy, CEQ, expectancy for improvement subscale (standardised); WAI-SR, Working Alliance Inventory Short Revised.

and the SDS, and increased in the QOLI from pre- to post-assessment and from pre- to 6-month follow-up assessment. Within-condition effect sizes revealed that most of these improvements were large in magnitude in both conditions.

\section{Additional results}

Means in participant-reported motivation and SE were similar across assessments for the conditions; see Table 4. Furthermore, means changed in the expected direction across assessments, except for motivation to change unhealthy lifestyle behaviours in the MI-CBT condition. Within-condition effect sizes in SE for performing activities despite psychiatric symptoms were of moderate to large magnitude across assessments for both conditions. However, effect sizes in motivation to change psychiatric symptoms and unhealthy lifestyle behaviours were mostly small, and mostly non-existent in SE for performing activities despite unhealthy lifestyle behaviours (Table 4). Observer-assessed motivation in terms of change talk was 8.8 and $7.5 \%$ in the CBT and MI-CBT conditions, respectively.

\section{Discussion}

The primary aim of the present pilot trial was to investigate the feasibility of MI-CBT in preparation for a future RCT designed to assess the effectiveness of MI-CBT in routine psychiatric care. A secondary aim was to conduct separate preliminary evaluations exploring the effects of CBT alone for anxiety disorders and depression and of MI-CBT for anxiety disorders, depression, and unhealthy lifestyle behaviours. It was expected that MI-CBT would prove as feasible as CBT and that participants in both conditions would show clinically meaningful improvements. 
Table 3. Means and within-condition Hedges' $g$ effect sizes in treatment outcome measures

\begin{tabular}{|c|c|c|c|c|c|c|}
\hline & \multirow[b]{2}{*}{ Condition } & \multicolumn{3}{|c|}{ Means } & \multirow{2}{*}{$\begin{array}{c}\text { Pre-post } \\
\text { effect size }[95 \% \mathrm{Cl}]\end{array}$} & \multirow{2}{*}{$\begin{array}{c}\text { Pre-6MFU } \\
\text { effect size }[95 \% \mathrm{Cl}]\end{array}$} \\
\hline & & Pre & Post & 6MFU & & \\
\hline \multirow[t]{2}{*}{ Psychiatric index } & CBT & $\begin{array}{l}\text { Mean }=0.16 \\
S D=0.73 \\
n=21\end{array}$ & $\begin{array}{l}\text { Mean }=-0.60 \\
S D=1.22 \\
n=10\end{array}$ & $\begin{array}{l}\text { Mean }=-0.71 \\
S D=1.41 \\
n=8\end{array}$ & $0.70[-0.25,1.72]$ & $0.70[-0.39,1.89]$ \\
\hline & MI-CBT & $\begin{array}{l}\text { Mean }=0.24 \\
S D=0.96 \\
n=27\end{array}$ & $\begin{array}{l}\text { Mean }=0.18 \\
S D=1.02 \\
n=18\end{array}$ & $\begin{array}{l}\text { Mean }=0.18 \\
S D=1.04 \\
n=16\end{array}$ & $0.06[-0.49,0.60]$ & $0.06[-0.51,0.62]$ \\
\hline \multirow[t]{2}{*}{ Lifestyle index } & CBT & $\begin{array}{l}\text { Mean }=-0.11 \\
S D=1.01 \\
n=19\end{array}$ & $\begin{array}{l}\text { Mean }=0.09 \\
S D=0.91 \\
n=10\end{array}$ & $\begin{array}{l}\text { Mean }=-0.36 \\
S D=0.81 \\
n=7\end{array}$ & $-0.19[-1.00,0.60]$ & $0.24[-0.41,0.93]$ \\
\hline & MI-CBT & $\begin{array}{l}\text { Mean }=0.02 \\
S D=0.80 \\
n=28\end{array}$ & $\begin{array}{l}\text { Mean }=0.06 \\
S D=1.01 \\
n=18\end{array}$ & $\begin{array}{l}\text { Mean }=0.29 \\
S D=0.86 \\
n=15\end{array}$ & $-0.04[-0.53,0.44]$ & $-0.31[-0.80,0.16]$ \\
\hline \multirow[t]{2}{*}{ GAD-7 } & CBT & $\begin{array}{l}\text { Mean }=12.67 \\
S D=5.82 \\
n=21\end{array}$ & $\begin{array}{l}\text { Mean }=5.80 \\
S D=4.59 \\
n=10\end{array}$ & $\begin{array}{l}\text { Mean }=6.38 \\
S D=4.87 \\
n=8\end{array}$ & $1.20[0.40,2.18]$ & $1.04[0.11,2.18]$ \\
\hline & MI-CBT & $\begin{array}{l}\text { Mean }=13.86 \\
S D=4.90 \\
n=28\end{array}$ & $\begin{array}{l}\text { Mean }=7.22 \\
S D=5.25 \\
n=18\end{array}$ & $\begin{array}{l}\text { Mean }=6.06 \\
S D=4.64 \\
n=16\end{array}$ & $1.25[0.65,1.94]$ & $1.55[0.77,2.47]$ \\
\hline \multirow[t]{2}{*}{ PHQ-9 } & CBT & $\begin{array}{l}\text { Mean }=16.76 \\
S D=4.63 \\
n=21\end{array}$ & $\begin{array}{l}\text { Mean }=6.10 \\
S D=4.31 \\
n=10\end{array}$ & $\begin{array}{l}\text { Mean }=7.38 \\
S D=4.27 \\
n=8\end{array}$ & $2.18[1.01,3.71]$ & $1.87[0.67,3.49]$ \\
\hline & MI-CBT & $\begin{array}{l}\text { Mean }=15.90 \\
S D=5.24 \\
n=28\end{array}$ & $\begin{array}{l}\text { Mean }=8.22 \\
S D=4.88 \\
n=18\end{array}$ & $\begin{array}{l}\text { Mean }=8.63 \\
S D=5.56 \\
n=16\end{array}$ & $1.45[0.80,2.21]$ & $1.28[0.54,2.13]$ \\
\hline \multirow[t]{2}{*}{ SDS } & CBT & $\begin{array}{l}\text { Mean }=19.91 \\
S D=4.00 \\
n=21\end{array}$ & $\begin{array}{l}\text { Mean }=8.20 \\
S D=7.36 \\
n=10\end{array}$ & $\begin{array}{l}\text { Mean }=6.13 \\
S D=3.91 \\
n=8\end{array}$ & $1.81[0.69,3.21]$ & $3.10[1.37,5.54]$ \\
\hline & MI-CBT & $\begin{array}{l}\text { Mean }=17.86 \\
S D=6.98 \\
n=28\end{array}$ & $\begin{array}{l}\text { Mean }=12.39 \\
S D=6.41 \\
n=18\end{array}$ & $\begin{array}{l}\text { Mean }=10.63 \\
S D=7.92 \\
n=16\end{array}$ & $0.78[0.11,1.50]$ & $0.92[0.21,1.70]$ \\
\hline \multirow[t]{3}{*}{ QOLI } & CBT & $\begin{array}{l}\text { Mean }=-0.27 \\
S D=1.72 \\
n=21\end{array}$ & $\begin{array}{l}\text { Mean }=1.58 \\
S D=1.81 \\
n=10\end{array}$ & $\begin{array}{l}\text { Mean }=0.88 \\
S D=2.04 \\
n=8\end{array}$ & $-0.96[-1.98,-0.08]$ & $-0.54[-1.47,0.28]$ \\
\hline & MI-CBT & $\begin{array}{l}\text { Mean }=-0.50 \\
S D=1.78\end{array}$ & $\begin{array}{l}\text { Mean }=0.74 \\
S D=1.89\end{array}$ & $\begin{array}{l}\text { Mean }=0.54 \\
S D=2.29\end{array}$ & $-0.65[-1.13,-0.20]$ & $-0.48[-0.91,-0.09]$ \\
\hline & & $n=28$ & $n=18$ & $n=16$ & & \\
\hline
\end{tabular}

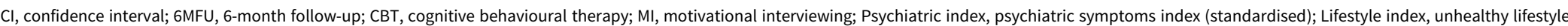
behaviors index (standardised); GAD-7, Generalized Anxiety Disorder Assessment-7; PHQ-9, Patient Health Questionnaire-9; SDS, Sheehan Disability Scale; QOLI, Quality of Life Inventory. 
Table 4. Means and within-condition Hedges' $g$ effect sizes for participant-reported motivation for behaviour change and self-efficacy

\begin{tabular}{|c|c|c|c|c|c|c|c|}
\hline & \multirow[b]{2}{*}{ Condition } & \multicolumn{3}{|c|}{ Means } & \multirow[b]{2}{*}{$\begin{array}{c}\text { Pre-session } 3 \text { effect size } \\
{[95 \% \mathrm{Cl}]}\end{array}$} & \multirow[b]{2}{*}{$\begin{array}{c}\text { Session 3-session } 6 \text { effect size } \\
{[95 \% \mathrm{Cl}]}\end{array}$} & \multirow[b]{2}{*}{$\begin{array}{c}\text { Pre-session } 6 \text { effect size } \\
{[95 \% \mathrm{Cl}]} \\
\end{array}$} \\
\hline & & Pre & Session 3 & Session 6 & & & \\
\hline \multirow[t]{2}{*}{ CQ psychiatric } & CBT & & $\begin{array}{l}\text { Mean }=24.06 \\
S D=3.79 \\
n=16\end{array}$ & $\begin{array}{l}\text { Mean }=25.69 \\
S D=3.17 \\
n=13\end{array}$ & & $-0.44[-0.93,0.01]$ & \\
\hline & MI-CBT & & $\begin{array}{l}\text { Mean }=24.29 \\
S D=3.27 \\
n=21\end{array}$ & $\begin{array}{l}\text { Mean }=24.82 \\
S D=2.68 \\
n=17\end{array}$ & & $-0.17[-0.56,0.21]$ & \\
\hline \multirow[t]{2}{*}{ CQ lifestyle } & CBT & & $\begin{array}{l}\text { Mean }=22.63 \\
S D=4.10 \\
n=16\end{array}$ & $\begin{array}{l}\text { Mean }=23.54 \\
S D=3.60 \\
n=13\end{array}$ & & $-0.22[-0.63,0.17]$ & \\
\hline & MI-CBT & & $\begin{array}{l}\text { Mean }=23.05 \\
S D=3.94 \\
n=21\end{array}$ & $\begin{array}{l}\text { Mean }=22.12 \\
S D=4.49 \\
n=17\end{array}$ & & $0.21[-0.29,0.72]$ & \\
\hline \multirow[t]{2}{*}{ SE psychiatric } & CBT & $\begin{array}{l}\text { Mean }=23.81 \\
S D=9.05 \\
n=21\end{array}$ & $\begin{array}{l}\text { Mean }=30.38 \\
S D=12.15 \\
n=16\end{array}$ & $\begin{array}{l}\text { Mean }=33.46 \\
S D=11.52 \\
n=13\end{array}$ & $-0.58[-1.03,-0.18]$ & & $-0.87[-1.64,-0.20]$ \\
\hline & MI-CBT & $\begin{array}{l}\text { Mean }=23.00 \\
S D=10.01 \\
n=28\end{array}$ & $\begin{array}{l}\text { Mean }=27.71 \\
S D=10.23 \\
n=21\end{array}$ & $\begin{array}{l}\text { Mean }=31.35 \\
S D=7.79 \\
n=17\end{array}$ & $-0.45[-0.78,-0.14]$ & & $-0.89[-1.53,-0.31]$ \\
\hline \multirow[t]{2}{*}{ SE lifestyle } & CBT & $\begin{array}{l}\text { Mean }=21.62 \\
S D=9.54 \\
n=21\end{array}$ & $\begin{array}{l}\text { Mean }=21.88 \\
S D=10.50 \\
n=16\end{array}$ & $\begin{array}{l}\text { Mean }=25.85 \\
S D=11.24 \\
n=13\end{array}$ & $-0.03[-0.35,0.30]$ & & $-0.38[-1.13,0.34]$ \\
\hline & MI-CBT & $\begin{array}{l}\text { Mean }=23.82 \\
S D=8.01 \\
n=28\end{array}$ & $\begin{array}{l}\text { Mean }=24.52 \\
S D=8.45 \\
n=21\end{array}$ & $\begin{array}{l}\text { Mean }=24.65 \\
S D=10.02 \\
n=17\end{array}$ & $-0.08[-0.47,0.30]$ & & $-0.09[-0.67,0.49]$ \\
\hline
\end{tabular}

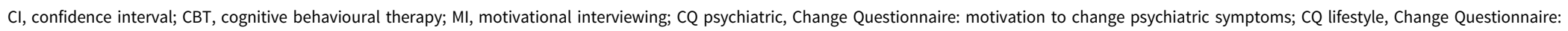

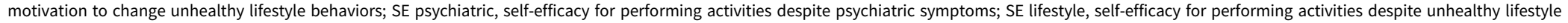
behaviours. 
The participants were recruited by the therapists, and the recruitment turned out to be rather slow. As suggested by anecdotal reports, this may have been due to therapist work stress, which may have led therapists to give less priority to recruitment. There was a high total drop-out rate of $42.9 \%$, which was much higher than in the other two studies (16.7 and 16.5\%, respectively) comparing CBT alone with MI-CBT in adult patients with anxiety disorders (Simpson et al., 2010; Westra et al., 2016). In neither the present study nor in the Simpson or Westra studies was the proportion of drop-out between conditions statistically different. The drop-out rate of the present study is more similar to the findings of the meta-analysis of drop-out in CBT by Fernandez and colleagues (Fernandez et al., 2015). In any case, the drop-out rate is a concern in terms of feasibility. There are at least two possible explanations. The first pertains to characteristics of participants; in the present study participants were recruited in routine psychiatric care, whereas in the Simpson and Westra studies, participants were recruited at a research clinic and from the general population, respectively. In fact, the psychiatric clinic where participants were treated in the present study exclusively deliver treatment to patients with 'complex' disorders; that is, patients with high symptom severity or co-morbidity or with previous treatment failures. A second explanation pertains to heavy participant burden in terms of extensive assessment, which involved both psychiatric symptoms and unhealthy lifestyle behaviours measures, and potential mediators of change, assessed at several time points.

Ratings of treatment credibility, expectancy for improvement, and working alliance were similar across the conditions. Thus, it does not appear that the integration of MI and CBT or the dual treatment focus negatively influenced participant perceptions of treatment or the relationship to the therapist. This finding provides some preliminary support of the feasibility of MI-CBT.

In general, and consistent with expectations, the evaluation of treatment effects suggested that participants in both conditions experienced large and clinically meaningful reductions in anxiety and depressive symptoms and functional impairment, and moderate to large increases in quality of life from pre- to post-assessment and from pre- to 6-month follow-up assessment. These results provide additional preliminary support of the feasibility of MI-CBT and warrant further evaluations of this treatment. However, in the psychiatric symptoms and unhealthy lifestyle behaviours indices, effect sizes varied from non-existent to moderate across conditions and assessments. There were moderate to large improvements in SE for performing activities despite psychiatric symptoms across assessments for both conditions. However, effect sizes in motivation to change psychiatric symptoms and unhealthy lifestyle behaviours were mostly small, and largely non-existent in SE for performing activities despite unhealthy lifestyle behaviours, across assessments for both conditions.

There are at least two possible explanations of the lack of effects on unhealthy lifestyle behaviours and observer-assessed and participant-reported motivation. First, therapists may not have targeted lifestyle behaviours, despite an explicit focus to do so in the MI-CBT condition, which may be due to a perceived lack of ability. Therapists in routine psychiatric care do not typically target lifestyle behaviours. However, the extent to which therapists focused on lifestyle behaviours was not assessed. Second, MI-CBT may not have been delivered adequately as therapists, contrary to expectations, reached beginning proficiency in MI on only some indicators of competence. This result is similar to the study by Simpson and colleagues (Simpson et al., 2010) in which therapists did not reach beginning proficiency on most indicators of competence in MI. In contrast, in the study by Westra and colleagues (Westra et al., 2016) only therapists demonstrating competence were allowed to enter the study and see participants. However, competence in the integration of MI and CBT was assessed neither in the present study nor the other studies, so it is not known to what extent therapists can actually provide skilful MI-CBT, rather than pure MI.

There are some limitations of the present study that should be taken into account when interpreting the results, and which may inform future studies. As already mentioned, 
therapists were not trained to competence in MI and CBT prior to entering the study; thus, treatment delivery may have been sub-optimal. Relatedly, fidelity was not assessed. Furthermore, the drop-out rate was high, indicating low feasibility in this respect. Finally, it cannot be ruled out that the differing assessment points after randomisation due to varying treatment length across participants to some extent could have influenced the results. Generalisability of the results may be limited to specialised psychiatric care settings.

\section{Conclusions}

In the present study, there were indications of feasibility of MI-CBT in some respects. However, because of insufficient participant recruitment and retention and therapist competence in MI, the study did not support the progression to an RCT designed to assess the effectiveness of MI-CBT in routine psychiatric care. Additional pilot studies are needed. Future pilot studies, and by extension RCTs, should ensure that these critical aspects are managed adequately, for example, by enabling an extended recruitment period and using procedures to ensure that therapists acquire competence.

The present pilot trial was only the second RCT to be conducted comparing CBT alone with MI-CBT in adult patients with anxiety disorders, and the first in a routine care setting using regular therapists. Moreover, it was the first trial to include participants with principal depression, a disorder that may be particularly apt for motivational work considering symptoms such as loss of interest and energy. In addition, it was one of few trials involving a dual treatment focus on both psychiatric disorders and unhealthy lifestyle behaviours. Given previous research of the benefits of combining MI and CBT, the paucity of studies integrating the treatments, and the potential benefit of simultaneous treatment of psychiatric disorders and unhealthy lifestyle behaviours, more research along these lines is warranted.

Acknowledgements. The authors would like to thank the participants and therapists at WeMind Psykiatri.

Financial support. This work was supported in part by the National Board of Health and Welfare (grant number 2.7-11638/ 2013).

Conflicts of interest. None.

Ethical statements. The authors have abided by the Ethical Principles of Psychologists and Code of Conduct as set out by the British Association for Behavioural and Cognitive Psychotherapies and the British Psychological Society. The study was approved by the Ethical Review Board in Stockholm, Sweden (2013/1566-31/3). Participants provided written informed consent.

Data availability statement. The data that support the findings of this study are available on request from the corresponding author. The data are not publicly available due to privacy or ethical restrictions.

Author contributions. AG and BB designed the study and wrote the initial draft. All authors conducted the statistical analyses, interpreted the results, and critically revised the manuscript. All authors read and approved the final manuscript.

\section{References}

American Psychiatric Association. (2000). Diagnostic and Statistical Manual of Mental Disorders: DSM-IV-TR (4th edn). Washington, DC, USA: American Psychiatric Association.

Baer, L., Brown-Beasley, M. W., Sorce, J., \& Henriques, A. I. (1993). Computer-assisted telephone administration of a structured interview for obsessive-compulsive disorder. American Journal of Psychiatry, 150, 1737-1738. https://doi. org/10.1176/ajp.150.11.1737

Bandura, A. (2006). A guide for constructing self-efficacy scales. In F. Pajares \& T. Urdan (eds), Self-Efficacy Beliefs of Adolescents (pp. 307-337). Charlotte, NC, USA: Information Age Publishing.

Batelaan, N. M., Seldenrijk, A., Bot, M., van Balkom, A. J., \& Penninx, B. W. (2016). Anxiety and new onset of cardiovascular disease: critical review and meta-analysis. British Journal of Psychiatry, 208, 223-231. https://doi.org/10. 1192/bjp.bp.114.156554 
Bohman, B., Rasmussen, F., \& Ghaderi, A. (2016). Development and psychometric evaluation of a context-based parental self-efficacy instrument for healthy dietary and physical activity behaviors in preschool children. International Journal of Behavioral Nutrition and Physical Activity, 13, 110. https://doi.org/10.1186/s12966-016-0438-y

Cicchetti, D. V. (1994). Guidelines, criteria, and rules of thumb for evaluating normed and standardized assessment instruments in psychology. Psychological Assessment, 6, 284-290. doi: http://dx.doi.org/10.1037/1040-3590.6.4.284

Craig, C. L., Marshall, A. L., Sjostrom, M., Bauman, A. E., Booth, M. L., Ainsworth, B. E., . . \& Oja, P. (2003). International physical activity questionnaire: 12-country reliability and validity. Medicine and Science in Sports and Exercise, 35, 1381-1395. https://doi.org/10.1249/01.MSS.0000078924.61453.FB

Craske, M. G., \& Barlow, D. H. (2008). Panic disorder and agoraphobia. In D. H. Barlow (ed), Clinical Handbook of Psychological Disorders: A Step-by-Step Treatment Manual (pp. 1-64). New York, USA: Guilford Press.

Cuijpers, P., Cristea, I. A., Karyotaki, E., Reijnders, M., \& Huibers, M. J. (2016). How effective are cognitive behavior therapies for major depression and anxiety disorders? A meta-analytic update of the evidence. World Psychiatry, 15, 245-258. https://doi.org/10.1002/wps.20346

Devilly, G. J., \& Borkovec, T. D. (2000). Psychometric properties of the Credibility/Expectancy Questionnaire. Journal of Behavior Therapy and Experimental Psychiatry, 31, 73-86. https://doi.org/10.1016/s0005-7916(00)00012-4

Dugas, M. J., \& Robichaud, M. (2007). Cognitive-Behavioral Treatment for Generalized Anxiety Disorder: From Science to Practice. New York, USA: Routledge.

Eldridge, S. M., Lancaster, G. A., Campbell, M. J., Thabane, L., Hopewell, S., Coleman, C. L., \& Bond, C. M. (2016). Defining feasibility and pilot studies in preparation for randomised controlled trials: development of a conceptual framework. PLOS One, 11, e0150205. https://.doi.org/10.1371/journal.pone.0150205

Fernandez, E., Salem, D., Swift, J. K., \& Ramtahal, N. (2015). Meta-analysis of dropout from cognitive behavioral therapy: magnitude, timing, and moderators. Journal of Consulting and Clinical Psychology, 83, 1108-1122. https://doi.org/10.1037/ cсp0000044

Fluharty, M., Taylor, A. E., Grabski, M., \& Munafo, M. R. (2017). The association of cigarette smoking with depression and anxiety: a systematic review. Nicotine and Tobacco Research, 19, 3-13. https://doi.org/10.1093/ntr/ntw140

Foa, E. B., Cashman, L., Jaycox, L., \& Perry, K. (1997). The validation of a self-report measure of posttraumatic stress disorder: the Posttraumatic Diagnostic Scale. Psychological Assessment, 9, 445-451. https://doi.org/10.1037/1040-3590.9.4.445

Foa, E. B., Hembree, E. A., \& Rothbaum, B. O. (2007). Prolonged Exposure Therapy for PTSD: Emotional Processing of Traumatic Experiences. Oxford, UK: Oxford University Press.

Franklin, M. E., \& Foa, E. B. (2008). Obsessive-compulsive disorder. In D. H. Barlow (ed), Clinical Handbook of Psychological Disorders: A Step-by-Step Treatment Manual (pp. 164-215). New York, USA: Guilford Press.

Fresco, D. M., Coles, M. E., Heimberg, R. G., Liebowitz, M. R., Hami, S., Stein, M. B., \& Goetz, D. (2001). The Liebowitz Social Anxiety Scale: a comparison of the psychometric properties of self-report and clinician-administered formats. Psychological Medicine, 31, 1025-1035. https://doi.org/10.1017/s0033291701004056

Frisch, M. B., Cornell, J., Villanueva, M., \& Retzlaff, P. J. (1992). Clinical validation of the Quality of Life Inventory: a measure of life satisfaction for use in treatment planning and outcome assessment. Psychological Assessment, 4, 92-101. https://doi.org/10.1037/1040-3590.4.1.92

Hatcher, R. L., \& Gillaspy, J. A. (2006). Development and validation of a revised short version of the Working Alliance Inventory (WAI-SR). Psychotherapy Research, 16, 12-25. https://doi.org/10.1080/10503300500352500

Houck, P. R., Spiegel, D. A., Shear, M. K., \& Rucci, P. (2002). Reliability of the self-report version of the panic disorder severity scale. Depression and Anxiety, 15, 183-185. https://doi.org/10.1002/da.10049

Kroenke, K., Spitzer, R. L., \& Williams, J. B. (2001). The PHQ-9: validity of a brief depression severity measure. Journal of General Internal Medicine, 16, 606-613. https://doi.org/10.1046/j.1525-1497.2001.016009606.x

Lakens, D. (2013). Calculating and reporting effect sizes to facilitate cumulative science: a practical primer for t-tests and ANOVAs. Frontiers in Psychology, 4, 863. https://doi.org/10.3389/fpsyg.2013.00863

Madson, M. B., Loignon, A. C., \& Lane, C. (2009). Training in motivational interviewing: a systematic review. Journal of Substance Abuse Treatment, 36, 101-109. https://doi.org/10.1016/j.jsat.2008.05.005

Marker, I., \& Norton, P. J. (2018). The efficacy of incorporating motivational interviewing to cognitive behavior therapy for anxiety disorders: a review and meta-analysis. Clinical Psychology Review, 62, 1-10. https://doi.org/10.1016/j.cpr.2018.04.004

Martell, C. R., Dimidjian, S., \& Herman-Dunn, R. (2010). Behavioral Activation for Depression: A Clinician's Guide. New York, USA: Guilford Press.

Meyer, T. J., Miller, M. L., Metzger, R. L., \& Borkovec, T. D. (1990). Development and validation of the Penn State Worry Questionnaire. Behaviour Research and Therapy, 28, 487-495. https://doi.org/ doi: 10.1016/0005-7967(90)90135-6

Miller, W. R., \& Johnson, W. R. (2008). A natural language screening measure for motivation to change. Addictive Behaviors, 33, 1177-1182. https://doi.org/10.1016/j.addbeh.2008.04.018

Miller, W. R., Moyers, T., Manuel, J., Christopher, P., \& Amrhein, P. (2007). Client Language Assessment in Motivational Interviewing (CLAMI). University of New Mexico: Center on Alcoholism, Substance Abuse and Addictions (CASAA). Albuquerque, NM, USA. 
Miller, W. R., \& Rollnick, S. (2013). Motivational Interviewing: Helping People Change (3rd edn). New York, USA: Guilford Press.

Miller, W. R., Yahne, C. E., Moyers, T. B., Martinez, J., \& Pirritano, M. (2004). A randomized trial of methods to help clinicians learn motivational interviewing. Journal of Consulting and Clinical Psychology, 72, 1050-1062. https://doi.org/10. 1037/0022-006X.72.6.1050

Moyers, T. B., Martin, T., Manuel, J. K., Miller, W. R., \& Ernst, D. (2010). Revised global scales: Motivational Interviewing Treatment Integrity 3.1.1 (MITI 3.1.1). Retrieved from: http://www.motivationalinterview.org/Documents/miti3_1.pdf

Muse, K., \& McManus, F. (2013). A systematic review of methods for assessing competence in cognitive-behavioural therapy. Clinical Psychology Review, 33, 484-499. https://doi.org/10.1016/j.cpr.2013.01.010

National Board of Health and Welfare (2011). National Guidelines for Methods of Preventing Disease. Stockholm, Sweden: National Board of Health and Welfare.

National Board of Health and Welfare (2018). National Guidelines for the Prevention and Treatment of Unhealthy Lifestyle Behaviors. Stockholm, Sweden: National Board of Health and Welfare.

Saunders, J. B., Aasland, O. G., Babor, T. F., de la Fuente, J. R., \& Grant, M. (1993). Development of the Alcohol Use Disorders Identification Test (AUDIT): WHO Collaborative Project on Early Detection of Persons with Harmful Alcohol Consumption-II. Addiction, 88, 791-804. https://doi.org/10.1111/j.1360-0443.1993.tb02093.x

Sepp, H., Ekelund, U., \& Becker, W. (2004). Survey Questions on Diet and Physical Activity in Adults. Report 21. Stockholm, Sweden: National Food Agency.

Sheehan, D. V. (1983). The Anxiety Disease. New York, USA: Charles Scribner and Sons.

Sheehan, D. V., Lecrubier, Y., Sheehan, K. H., Amorim, P., Janavs, J., Weiller, E., . . \& Dunbar, G. C. (1998). The MiniInternational Neuropsychiatric Interview (M.I.N.I.): the development and validation of a structured diagnostic psychiatric interview for DSM-IV and ICD-10. Journal of Clinical Psychiatry, 59, suppl 20, 22-33; quiz, 34-57.

Simpson, H. B., Zuckoff, A. M., Maher, M. J., Page, J. R., Franklin, M. E., Foa, E. B., . . \& Wang, Y. (2010). Challenges using motivational interviewing as an adjunct to exposure therapy for obsessive-compulsive disorder. Behaviour Research and Therapy, 48, 941-948. https://doi.org/10.1016/j.brat.2010.05.026

Spitzer, R. L., Kroenke, K., Williams, J. B., \& Lowe, B. (2006). A brief measure for assessing generalized anxiety disorder: the GAD-7. JAMA Internal Medicine, 166, 1092-1097. https://doi.org/10.1001/archinte.166.10.1092

Springer, K. S., Levy, H. C., \& Tolin, D. F. (2018). Remission in CBT for adult anxiety disorders: a meta-analysis. Clinical Psychology Review, 61, 1-8. https://doi.org/10.1016/j.cpr.2018.03.002

Turk, C. L., Heimberg, R. G., \& Magee, L. (2008). Social anxiety disorder. In D. H. Barlow (ed), Clinical Handbook of Psychological Disorders: A Step-by-Step Treatment Manual (pp. 123-163). New York, USA: Guilford Press.

Westra, H. A. (2012). Motivational Interviewing in the Treatment of Anxiety. New York, USA: Guilford Press.

Westra, H. A., Constantino, M. J., \& Antony, M. M. (2016). Integrating motivational interviewing with cognitive-behavioral therapy for severe generalized anxiety disorder: an allegiance-controlled randomized clinical trial. Journal of Consulting and Clinical Psychology, 84, 768-782. https://doi.org/10.1037/ccp0000098

Zschucke, E., Gaudlitz, K., \& Strohle, A. (2013). Exercise and physical activity in mental disorders: clinical and experimental evidence. Journal of Preventive Medicine and Public Health, 46, suppl 1, S12-21. https://doi.org/10.3961/jpmph.2013.46.S.S12

Cite this article: Ghaderi A, Rosendahl I, and Bohman B (2022). Integrating motivational interviewing with cognitive behavioural therapy for anxiety disorders, depression and co-morbid unhealthy lifestyle behaviours: a randomised controlled pilot trial. Behavioural and Cognitive Psychotherapy 50, 74-88. https://doi.org/10.1017/S1352465821000345 